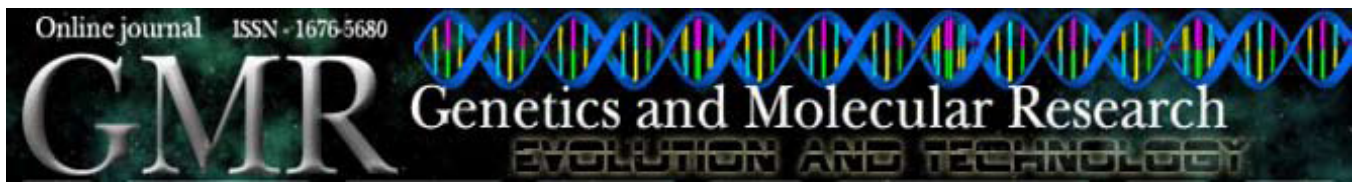

\title{
Overexpression of type-A rice response regulators, OsRR3 and OsRR5, results in lower sensitivity to cytokinins
}

\author{
X. Cheng*, H. Jiang*, J. Zhang, Y. Qian, S. Zhu and B. Cheng \\ School of Life Science, Anhui Agricultural University, Hefei, Anhui, China \\ *These authors contributed equally to this study. \\ Corresponding author: B. Cheng \\ E-mail: beijiucheng@ahau.edu.cn
}

Genet. Mol. Res. 9 (1): 348-359 (2010)

Received November 13, 2009

Accepted December 8, 2009

Published March 2, 2010

\begin{abstract}
Response regulators are part of a two-component regulatory system. The type-A Arabidopsis response regulators act as negative regulators. To understand the function of type-A response regulators in the model monocot plant, rice (Japonica cultivar-group: Zhonghua11), we overexpressed two type-A OsRR genes, OsRR3 and OsRR5 (pACT1:OsRR3 and pACT1:OsRR5). We hoped to gain insight into their molecular function in cytokinin-signaling pathways. Both OsRR3 and OsRR5 overexpressors had longer roots and more lateral roots compared with Zhonghua11, when treated with exogenous cytokinin. Using callus formation and chlorophyll content assays, we found that Zhonghua11 was more sensitive to cytokinin compared with other cultivars of rice, expressing high transcript levels of OsRR3 and OsRR5. The expression of most type-A OsRR genes was repressed by OsRR3 and OsRR5 overexpression. However, semi-quantitative RTPCR showed that three type-A OsRR genes showed increased expression. Our results suggest that both OsRR3 and OsRR5 mainly act as negative regulators of cytokinin signaling, as indicated by the reduced sensitivity of OsRR3 and OsRR5 overexpressors to exogenous cytokinins.
\end{abstract}

Key words: Cytokinin; Response regulator; Rice; RT-PCR 


\section{INTRODUCTION}

Cytokinins are plant hormones that regulate various growth and physiological processes, such as cell division, apical dominance, cell proliferation, chloroplast biogenesis, vascular differentiation, root and leaf differentiation, photomorphogenic development, and inhibition of leaf senescence (Mok and Mok, 2001). Genetic and molecular research in plants has suggested the involvement of a two-component signaling system in cytokinin signal perception and transduction, which are used by prokaryotic and eukaryotic organisms (Stock et al., 2000). The simple two-component system involves a His kinase (HK) sensor that perceives the signal and a response regulator (RR) that mediates the output of the pathway. The HK sensor autophosphorylates a His residue in response to an output signal, and the phosphate group is transferred to a conserved Asp residue in the receiver domain of the response regulator. The downstream signaling of the pathway is then modulated by the phosphorylated response regulator. The cytokinin multistep two-component signaling system comprises sensor histidine kinase proteins, histidine phosphotransfer proteins $(\mathrm{HPt})$, and response regulator proteins. In this pathway, signals are transferred from a His in the receptor kinase to an Asp in the response regulator via His-Asp-His-Asp phosphotransfer reactions between alternating His and Asp residues (Kakimoto, 2003; Muller and Sheen, 2007).

In Arabidopsis thaliana, cytokinin independent 1 (CKI1) was first isolated in an activation-tagging screen for hypocotyls that produce calli in the absence of exogenous cytokinin. As CKI1 did not require cytokinin for its activity, it was not thought to be a cytokinin receptor (Kakimoto, 1996). The cytokinin receptors cytokinin response 1 (CRE1), wooden leg 1 (WOL), Arabidopsis histidine kinase 4 (AHK4), and its homologs AHK2 and AHK3 are similar to bacterial His sensor hybrid kinases, which include a ligandbinding site, a His kinase domain, and a receiver domain, and were suggested to be receptors for cytokinins by yeast and Escherichia coli systems (Inoue et al., 2001; Suzuki et al., 2001; Ueguchi et al., 2001a,b). Loss-of-function mutations of the cytokinin receptor CRE1 exhibited reduced cytokinin sensitivity, and overexpression of AHK2 in Arabidopsis protoplasts enhanced the cytokinin-induced expression from the ARR6 promoter (Inoue et al., 2001; Hwang and Sheen, 2001). Analysis of ahk2, ahk3, and ahk4 with double mutants showed reduced response to cytokinin, and triple mutants where all three cytokinin receptors were disrupted indicated that they possess distinct overlapping functions in shoot, root, and seed growth (Higuchi et al., 2004; Nishimura et al., 2004). All plant HKs contain a C-terminal receiver domain and a conserved transmitter domain, which includes characteristic sequence motifs and a conserved His residue (West and Stock, 2001). In a recent study, three Arabidopsis HK genes, three maize HK genes, and five rice HK genes have been identified (Inoue et al., 2001; Yonekura-Sakakibara et al., 2004). In response to cytokinin binding, HKs autophosphorylate on a conserved His residue and then transfer the phosphate group to response regulators via an intermediate set of histidine phosphotransfer proteins (Suzuki et al., 1998; Hutchison et al., 2006). The majority of HPs act as redundant, positive regulators of cytokinin signaling and affect many aspects of plant development (Hutchison et al., 2006). In addition, physiological and biochemical analyses have shown that most AHPt proteins are capable of interacting with the hybrid kinase and response regulator (Urao et al., 1998). 
The typical response regulators in Arabidopsis are classified into type-A and typeB (Hwang et al., 2002). The type-A response regulators are relatively small, containing a receiver domain along with short $\mathrm{N}$ - and $\mathrm{C}$-terminal extensions (Imamura et al., 1998; Brandstatter and Kieber, 1998). The type-B response regulators have a receiver domain, an extensive $\mathrm{C}$-terminal extension involved in transcription regulation (Sakai et al., 1998; Lohrmann et al., 1999), and a type-B signature GARP motif of about 60 amino acids that is related to the Myb repeat of transcription factors (Sakai et al., 2000). Expression is rapidly induced in response to exogenous cytokinin in the type-A ARR genes. In contrast, the steady-state levels of type-B ARR transcripts are not affected by cytokinin or other plant hormones (Brandstatter and Kieber, 1998; Taniguchi et al., 1998; Lohrmann and Harter, 2002). Overexpression of various type-A ARRs in Arabidopsis protoplasts repressed the ability of cytokinin to induce the ARR6 promoter, indicating that type-A ARRs are transcriptional repressors (Hwang and Sheen, 2001). Overexpression of ARR8 repressed shoot regeneration and greening of calli, and overexpression of ARR15 resulted in reduced sensitivity to cytokinin (Osakabe et al., 2002; Kiba et al., 2003). Overexpression of ARR7 showed that it is a negative regulator of early cytokinin-regulated genes and mainly acts as a transcriptional repressor (Lee et al., 2007). In rice, type-A genes have been isolated and characterized (Sakakibara et al., 1998). Overexpression of OsRR6 displayed altered morphologies and changes in cytokinin metabolism (Hirose et al., 2008).

The response regulators involved in cytokinin signaling have been reported in the Gramineae, but knowledge of type-A response regulators in rice is very limited compared with Arabidopsis. To better understand the role of type-A OsRRs in cytokinin signaling, we overexpressed OsRR3 and OsRR5, two type-A OsRRs, in rice and investigated which genes are regulated by OsRR3 and OsRR5 in response to cytokinin.

\section{MATERIAL AND METHODS}

\section{Plasmid construction and rice transformation}

Total RNA was extracted from the Japonica cultivar-group: Zhonghua11 (ZH11) using the RNAiso reagent (TaKaRa Biotechnology, Japan). After RNase-free DNase (TaKaRa Biotechnology) treatment, purified RNA was reverse transcribed using the Access RT-PCR (reverse transcription-polymerase chain reaction) system (Promega, USA) to obtain first-strand cDNA. The OsRR3 and OsRR5 sequences were amplified from cDNA with the following primers: 5-GCTCTAGATGTCGACGAAGACAGTGCC-3 and 5-AACTGCAGCAAAGT CATTTCATGATGACGC-3 for OsRR3, and 5-GCTCTAGATCTGATGGCCACCTGC-3 and 5-GCTCTAGATCACCGGAGGACGCGGC-3 for OsRR5. The PCR products were subcloned into the pMD18-T vector (TaKaRa Biotechnology). The fragments with the coding regions of OsRR3 and OsRR5 were inserted into the pXQACT vector, after removing the GUS gene, under the control of the pACT1 promoter. The resulting pACT1:OsRR3 and pACT1:OsRR5 constructs were introduced into ZH11 by Agrobacterium-mediated transformation. Rice plants were cultivated in the experimental field at Anhui Agricultural University in the natural growing season. For the analysis of cytokinin-responsive genes in rice, we screened T1-generation transgenic rice using the selective marker NPTII, which has resistance to G418. The haploid T2-generation transgenic rice plants were then selected by DNA hybridization to eliminate 
polyploidy transgenic rice (data not shown). Finally, progeny from identified T3 homozygous transformants for OsRR3 and OsRR5 overexpression was produced and used in subsequent experiments. ZH11 was used as the wild-type line for all experiments.

\section{Seedling hormone response assays}

Rice seeds were sterilized with $0.1 \% \mathrm{HgCl}_{2}$ for $1 \mathrm{~h}$ and washed with distilled water before soaking overnight in distilled water. The seeds were transferred to a nylon net floating on sterilized water. For hormone sensitivity assay, seeds were grown on plates at $23^{\circ} \mathrm{C}$ in a culture room with a 16-h photoperiod for 6 days, then transferred to plates containing either different concentrations of 2,4$\mathrm{D}$ (2,4-dichlorophenoxy acetic acid), 6-BA (6-benzyladenine acid), and abscisic acid (ABA) in $0.1 \%$ DMSO (dimethylsulfoxide), respectively, or an equal volume of DMSO as a control for another 10 days.

\section{Hormone treatment}

Rice seeds were grown on germination agar plates containing N6 media with vitamins, $3 \%$ sucrose, $0.03 \%$ casein enzymatic hydrolysate, $0.3 \%$ agar, $\mathrm{pH} 5.9$, at $28^{\circ} \mathrm{C}$ in a culture room with constant light. To test the hormone response of rice calli, seeds were grown on N6 media for 7 days and then transferred to sterilized N6 media agar plates containing different concentrations of 2,4-D and 6-BA for callus induction. After 3 weeks under constant light, rice calli were photographed with a digital camera.

\section{Chlorophyll content assays}

Seeds were grown on plates at $23^{\circ} \mathrm{C}$ with a 16 -h photoperiod for 30 days. For chlorophyll assays, seedlings were transferred to new plates supplemented with different concentrations of 6-BA and incubated in the dark at $23^{\circ} \mathrm{C}$ for either 2 or 4 days. To assess dark-induced leaf senescence, full leaves were detached from the plants and the chlorophylls were extracted with methanol. Chlorophyll content was then measured spectrophotometrically (Porra et al., 1989).

\section{Semiquantitative RT-PCR analysis}

Seeds were germinated on plates at $37^{\circ} \mathrm{C}$ for 2 days and then transferred to new plants in a nutrient solution at $28^{\circ} \mathrm{C}$ in a culture room under a 16 -h light and a 8 -h dark photoperiod for an additional 15 days. For cytokinin assays, the seedlings were transferred to a nutrient solution supplemented with $1 \mu \mathrm{M}$ 6-BA in $0.1 \%$ DMSO or an equal volume of DMSO as a control for $2 \mathrm{~h}$ and then harvested. Total RNAs were extracted from roots and leaves of seedlings with the RNAiso reagent (TaKaRa Biotechnology) according to manufacturer instructions. cDNAs were generated from the RNA with the Access RT-PCR system (Promega, USA). To examine the expression patterns of OsRR genes, sets of specific primers for the corresponding genes were designed. The rice actin1 gene was used as an internal control to normalize for variance in the quality of RNA and the amount of input cDNA. Reactions were performed using Taq polymerase (TaKaRa Biotechnology) on a thermal cycler (Tpersonal 48; Biometra, Germany). A $3-\mu \mathrm{L}$ aliquot of the reaction mix was separated on $1 \%(\mathrm{w} / \mathrm{v})$ agarose gels and stained with ethidium bromide. Two independent experiments were performed with consistent results. 


\section{RESULTS}

\section{Validation of T3 transgenic rice plants}

Transgenic plants overexpressing the OsRR 3 and OsRR5 genes were generated, and the expression of the OsRR3 and OsRR5 genes was examined to determine the levels of OsRR3 and OsRR5 in the transgenic plants. RT-PCR analysis showed that plants harboring pACT1:OsRR3 and pACT1:OsRR5 had significantly increased levels of OsRR3 and OsRR5 transcripts. All T3 transgenic rice plants exhibited higher expression of OsRR3 or OsRR5 compared with ZH11 (Figure 1). Transgenic lines 3-04-1, 3-08-1, and 5-03-1, 5-05-1, and 5-06-1 were used in all subsequent experiments based on their high OsRR3 or OsRR5 expression levels.

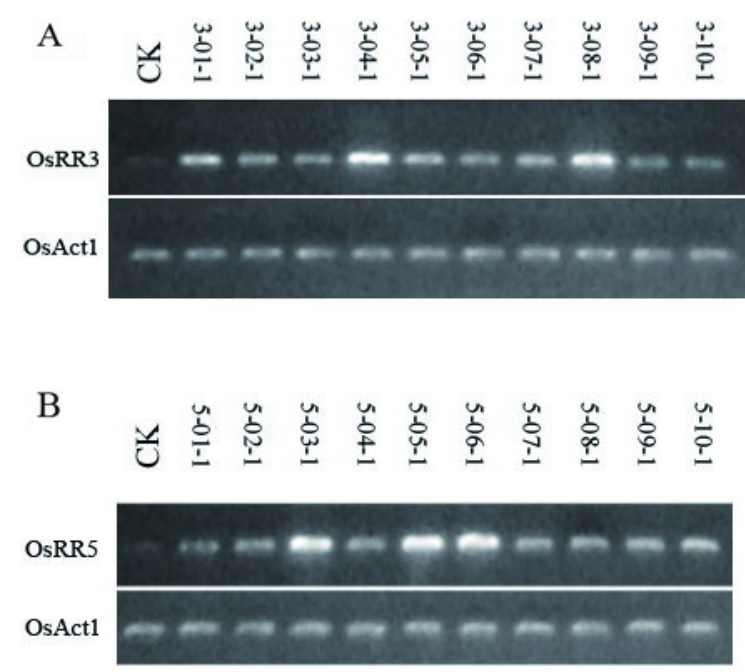

Figure 1. Semi-quantitative RT-PCR identification of transgenic rice. Total RNAs were prepared from leaves of transgenic plants and ZH11 (labeled CK). OsAct1 was amplified as a control. A. PCR identification of OsRR3 overexpression in transgenic rice. B. PCR identification of OsRR5 overexpression in transgenic rice.

\section{Rice seedlings overexpressing OsRR3 and OsRR5 are less sensitive to cytokinin inhibition of root elongation and lateral root formation}

The two transgenic rice lines constitutively overexpressing OsRR3 and OsRR5 (pACT1:OsRR3 and pACT1:OsRR5) looked similar to cultivated species ZH11, in terms of leaf initiation, formation of leaves, roots, flowers, and seeds. To assess the roles of the two type-A OsRRs in the cytokinin response pathway, we examined root elongation and lateral root formation in response to exogenous hormone addition. We compared root elongation of ZH11 with pACT1:OsRR3 and pACT1:OsRR5 seedlings across a range of hormone concentrations between 0 and $10 \mu \mathrm{M}$ (Figure 2). In the absence of exogenous hormone, the roots of ZH11, pACT1:OsRR3, and pACT1:OsRR5 were the same. The roots of all three kinds of rice were not affected until the hormone concentration reached $10 \mathrm{nM}$ (Figure 2). With further increase in BA concentration, primary root elongation decreased sharply, with a half-maximal inhibition at 0.3 
$\mu \mathrm{M}$ (Figure $2 \mathrm{~A}$ ). At higher concentrations $(>5 \mu \mathrm{M})$, primary root elongations of the three kinds of rice were similar (Figure 2). At concentrations of BA of 0.01-5 $\mu \mathrm{M}, \mathrm{pACT} 1$ :OsRR3 and pACT1:OsRR5 plants had longer roots compared to ZH11. This showed that pACT1:OsRR3 and pACT1:OsRR5 were less sensitive to cytokinin compared to ZH11 (Figure 2A). However, the root growth responses of pACT1:OsRR3 and pACT1:OsRR5 to varying concentrations of other plant hormones (2,4-D or ABA) were similar to that of $\mathrm{ZH} 11$, indicating that resistance to root elongation inhibition in both pACT1:OsRR3 and pACT1:OsRR5 are cytokinin dependent (Figure 2B and C). Formation of lateral roots is inhibited by cytokinin in plants (Werner et al., 2003). In the lateral root formation assay, we measured the number of lateral roots on ZH11, pACT1:OsRR3, and pACT1:OsRR5 with a range of BA concentrations between 0 and $10 \mu \mathrm{M}$. Lateral root formation decreased sharply between 0.01 and $1 \mu \mathrm{M}$ in ZH11 compared with pACT1:OsRR3 and pACT1:OsRR5 (Figure 2D). Overall, the results were similar to those observed in the root elongation assay. These results showed that high levels of both OsRR3 and OsRR5 expression reduce sensitivity to exogenous application of cytokinin.


Figure 2. Analysis of root elongation and lateral root formation during overexpression of OsRR3 and OsRR5 in transgenic rice and ZH11 (CK) in the presence of exogenous hormones. A., B., C. Seeds were grown on plates supplemented with the specified concentrations of BA, 2,4-D, ABA, or DMSO control, under a 16-h photoperiod at $23^{\circ} \mathrm{C}$. Error bars represent $\mathrm{SE}(\mathrm{N}=30)$. D. Seeds were grown on plates supplemented with the specified concentrations of BA control under a 16 -h photoperiod at $23^{\circ} \mathrm{C}$. The total number of lateral roots was determined at 15 days. Error bars represent $\mathrm{SE}(\mathrm{N}=30)$. E. Phenotypes of roots for 16-day-old seedlings of (from left to right) ZH11, pACT1:OsRR3, and pACT1:OsRR5 treated with $0.5 \mu \mathrm{M}$ BA under a 16-h photoperiod. 


\section{Callus formation of pACT1:OsRR3 and pACT1:OsRR5 is less sensitive to cytokinin}

Cytokinin promotes cell division and initiates shoots in concert with auxin in cultured plant tissues (Miller et al., 1955). To investigate the cytokinin responses of OsRR3 and OsRR5 transgenic plants, we examined callus formation in ZH11, pACT1:OsRR3, and pACT1:OsRR5 in response to various concentration of the cytokinin 6-BA and the auxin 2,4-D. ZH11 explants formed green shoots at high cytokinin:auxin ratios. At low cytokinin:auxin ratios, calli were observed. With increasing cytokinin:auxin ratios, larger calli were formed and green shoots were longer (Figure 3A). Both pACT1:OsRR3 and pACT1:OsRR5 formed smaller calli and shorter shoots with concentrations of hormones that were able to induce ZH11 calli (Figure 3B and C). The decrease in sensitivity to hormones of pACT1:OsRR3 and pACT1:OsRR5 in callus and shoot formation showed that both OsRR3 and OsRR5 act as negative regulators of the cytokinin-signaling system.
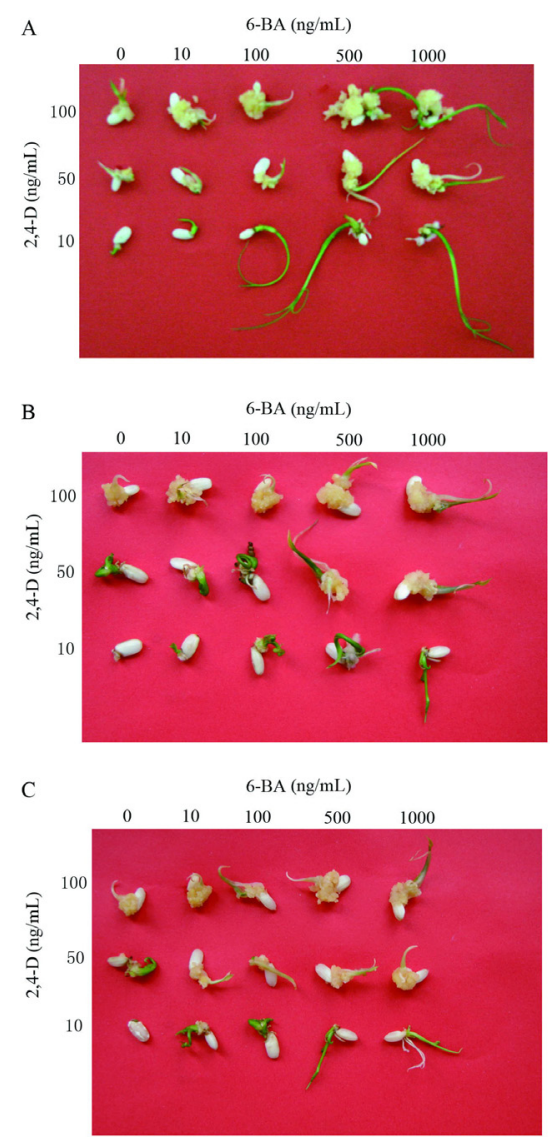

Figure 3. Callus formation of ZH11, pACT1:OsRR3, and pACT1:OsRR5. Rice seeds were grown on N6 media containing specified concentrations of 2,4-D and 6-BA. After 4 weeks in culture, the induced calli were arranged and photographed. A. ZH11. B. pACT1:OsRR3. C. pACT1:OsRR5. 


\section{Reduced chlorophyll content in pACT1:OsRR3 and pACT1:OsRR5}

Cytokinins take part in most aspects of plant growth and development, including leaf senescence. Cytokinins are known to delay leaf senescence (Gan and Amansino, 1995). For this reason, we measured chlorophyll content with various concentrations of BA to test the effects of exogenous cytokinin on the senescence process. Without dark-induced treatment, the chloroplast levels of ZH11, pACT1:OsRR3 and pACT1:OsRR5 were similar. After 2 days of dark-induced senescence, the leaf chloroplast levels of pACT1:OsRR3 and pACT1:OsRR5 plants were more reduced compared with ZH11 at the same concentrations of BA. A similar tendency was found after an additional 2 days of dark-induced senescence (Figure 4). Leaf senescence induced by the dark was delayed by increasing BA concentration, but the chlorophyll content was more reduced in pACT1:OsRR3 and pACT1:OsRR5 with increasing BA concentrations compared with ZH11. These data also confirm that overexpression of OsRR3 and OsRR5 reduces sensitivity to exogenous cytokinins.

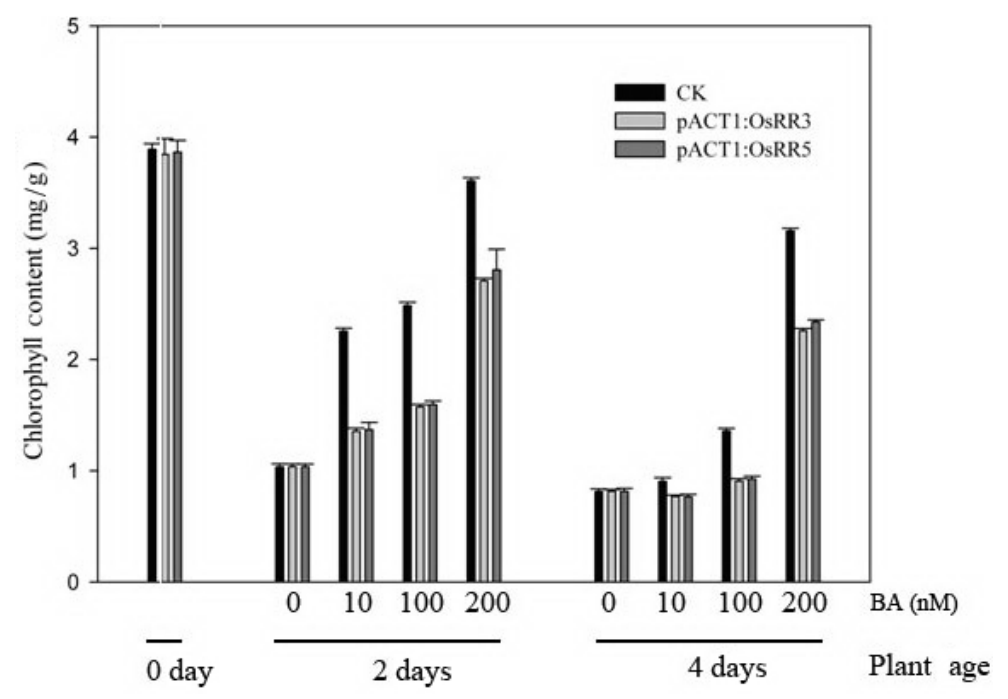

Figure 4. pACT1:OsRR3 and pACT1:OsRR5 show accelerated leaf senescence. Full leaves of 30-day-old ZH11 (CK), pACT1:OsRR3 and pACT1:OsRR5 plants were cultured in $0.1 \%$ DMSO containing given concentrations of BA for either 2 or 4 days in the dark. Chlorophyll content was determined spectrophotometrically as described in the Methods. Results are pooled from four independent experiments $(\mathrm{N}=15)$.

\section{Expression of OsRR genes during cytokinin treatment}

The expression of the OsRR genes was examined by RT-PCR. The expression of most type-A and type-B OsRR genes was detected in leaves and roots at different levels, except for OsRR11, which was expressed only in roots. However, three type-A OsRR genes: OsRR8, OsRR12, and OsRR 13, and one type-B OsRR gene, OsRR19, were not detected in leaves and roots. The expression of type-B OsRR genes was higher in both leaves and roots compared with type-A OsRR genes (Figure 5A and C). The expressions of most type-A ARR genes increased 
rapidly in response to exogenous cytokinin (D'Agostino et al., 2000), and thus, we analyzed the expression of OsRR genes after cytokinin treatment for $2 \mathrm{~h}$. The results showed different OsRR expression patterns in ZH11, pACT1:OsRR3, and pACT1:OsRR5. However, the results for pACT1:OsRR3 and pACT1:OsRR5 were similar (Figure 5B and D). After exogenous cytokinin 6-BA treatment, the expressions of most type-A OsRR genes increased in ZH11, but type-B OsRR genes were not affected. In contrast, the expression of most type-A OsRR genes decreased or did not increase in the two transgenic plants, with the exception of the OsRR2, OsRR4, and OsRR6; the transcripts of these three type-A OsRR genes accumulated. Similar to ZH11, type-B OsRR genes were not affected. The results suggest that OsRR3 and OsRR5 are primary cytokinin response genes, functioning as negative regulators of cytokinin signaling.

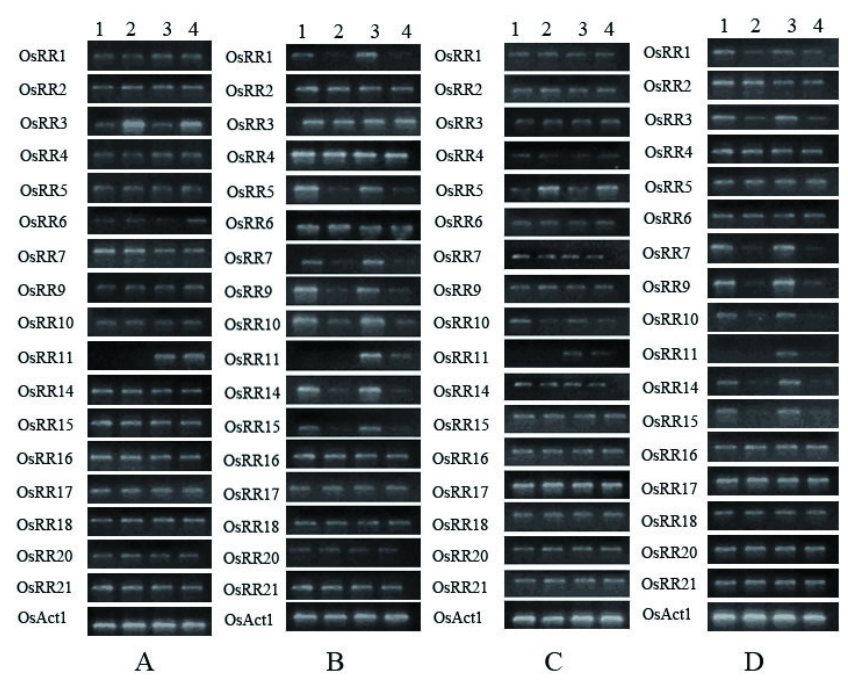

Figure 5. Expression pattern of OsRR genes. RNAs were isolated from roots and leaves from ZH11, pACT1:OsRR3, and pACT1:OsRR5 growing in nutrient medium or with $1 \mu \mathrm{M}$ 6-BA. OsACT1 was used as a template control. A. Expression of OsRR genes without hormone. Lane 1 = Leaf of ZH11; lane 2 = leaf of pACT1:OsRR3; lane $3=$ root of ZH11; lane 4 = root of pACT1:OsRR3. B. Expression of OsRR genes treated with $1 \mu \mathrm{M} 6$-BA for $2 \mathrm{~h}$. Lane 1 = Leaf of ZH11; lane 2 = leaf of pACT1:OsRR3; lane 3 = root of ZH11; lane 4 = root of pACT1:OsRR3. C. Expression of OsRR genes without hormone. Lane 1 = Leaf of ZH11; lane 2 = leaf of pACT1:OsRR5; lane 3 $=\operatorname{root}$ of ZH11; lane 4 = root of pACT1:OsRR5. D. Expression of OsRR genes treated with $1 \mu \mathrm{M} 6-\mathrm{BA}$ for $2 \mathrm{~h}$. Lane 1 = Leaf of ZH11; lane 2 = leaf of pACT1:OsRR5; lane 3 = root of ZH11; lane 4 = root of pACT1:OsRR5.

\section{DISCUSSION}

The components of the cytokinin-signaling system comprise histidine kinases, histidine phosphotransferase proteins, and response regulators. Various genetic, molecular biologi$\mathrm{cal}$, and biochemical studies have suggested that type-A Arabidopsis response regulators are negative regulators in cytokinin signaling; however, studies of cytokinin signaling in rice are limited. To gain new insights into the function of type-A OsRRs in cytokinin signaling, OsRR3 and OsRR5, two type-A OsRRs, were overexpressed in rice under the control of the rice actin1 
(pACT1) promoter. This promoter produces much higher levels of constitutive expression in rice compared with the cauliflower mosaic virus $35 \mathrm{~S}$ promoter (Sentoku et al., 2000).

There are many reports of the expression of cytokinin-inducible type-A ARR genes in Arabidopsis. Overexpression of ARR4 in cultured stems of transgenic plants promoted shoot formation in the presence of cytokinin, and overexpression of ARR8 repressed shoot formation and greening of calli (Osakabe et al., 2002). Root elongation was enhanced by ARR15 overexpression (Kiba et al., 2003). Overexpression ARR7 led to reduced sensitivity to cytokinin (Lee et al., 2007). Our results showed that overexpression of OsRR3 and OsRR5 causes reduced sensitivity to cytokinin in terms of root elongation, lateral root formation, callus formation, and darkinduced leaf senescence. OsRRs are cytokinin response genes, but the two overexpressing plants were not affected by 2,4-D and ABA in the root elongation assays. In addition, overexpression of OsRR6 alters rice morphology (abnormal flowers and sterility) and cytokinin metabolism (Hirose et al., 2007). Overexpression of ARR4 and ARR8 alters cytokinin responses differentially, in terms of flowering time and longer stems, compared with control plant (Osakabe et al., 2002). However, in our experiments, transgenic rice showed normal growth compared with ZH11, and showed no changes in morphogenesis, such as formation of leaves, roots, stem, flowers, flowering time, and callus formation of adult plants. We hypothesize that type-A RRs may not exhibit the same cytokinin-signaling regulation in all conditions, and may use different pathways to affect upstream and downstream development in the cytokinin-signaling system.

In our experiments, both pACT1:OsRR3 and pACT1:OsRR5 showed similar results in different experiments. According to protein sequence analysis, we found that the OsRR3 and OsRR5 proteins have high homology, with highly conserved Lys and Asp residues (D-D$\mathrm{K})$ in the receiver domain. By phylogenetic analysis, we know that OsRR3 and OsRR5 have a highly homologous phylogenetic relationship (Jain et al., 2006). Thus, we hypothesize that OsRR3 and OsRR5 have similar functions in the cytokinin-signaling system.

The mRNA of most type-AARRs, ZmRRs, and OsRRs rapidly accumulate after cytokinin treatment (Sakakibara et al., 1998; Asakura et al., 2003; Giulini et al., 2004; Hirose et al., 2007). In our study, the expression of OsRR genes in the two overexpressing plants was the same as in the wild-type ZH11 in the absence of cytokinin. More than six RR genes are induced by cytokinin, yet only three of the induced genes are validated by the results of Hirose et al. (2008). This result may be due to different environmental factors and different tissues used for the expression of these genes in this study. We found that OsRRs expressions are different in leaves and roots, and that the expression of the same OsRR gene in leaf and root is different, indicating that cytokinin signaling in rice may have overlapping functions. After cytokinin treatment, the expression of three of the 16 type-A OsRR genes was increased, and that of nine type-A OsRR genes was reduced, while the remaining 3 type-A genes were not detected. Overexpression of OsRR3 and OsRR5 caused transcription repression but also activated type-A OsRRs, such as OsRR2, OsRR4, and OsRR6. One possible explanation is that overexpression of OsRR3 and OsRR5 mainly participates in the repression of the transcription of other type-A OsRRs, providing a negative feedback mechanism. Most of the OsRR genes were expressed at different levels in leaves and roots without exogenous cytokinin treatment. The transcript levels of type-B OsRRs are relatively high compared with type-A OsRR genes, and the expression of OsRR11 was not found in leaves. OsRR8, OsRR12 and OsRR13 transcripts were not found in either leaves or roots. In contrast, OsRR8, OsRR12, and OsRR13 were expressed in flowers and panicles (Ito and Kurata, 2006). These results suggest that OsRR genes exhibit organ-specific expression and differential expression patterns. The 
type-B RRs are the transcription factors that respond to cytokinins (Sakai et al., 2000). We found that the expression levels of type-B OsRRs did not change in response to exogenous cytokinin treatment; thus, we think that type-A OsRRs may represent the primary cytokinin response genes. The data presented here indicate that the cytokinin-signaling system in rice is a complex-signaling pathway that involves a degree of cross-talk.

\section{ACKNOWLEDGMENTS}

Research supported by the National Natural Science Foundation of China (\#10675002) and National High-Tech Research and Development Program (863 Program) (\#2008AA10Z408). We thank members of the Key Laboratory of Crop Biotechnology of Anhui Province and Guo Wei for assistance in conducting these experiments.

\section{REFERENCES}

Asakura Y, Hagino T, Ohta Y, Aoki K, et al. (2003). Molecular characterization of His-Asp phosphorelay signaling factors in maize leaves: implications of the signal divergence by cytokinin-inducible response regulators in the cytosol and the nuclei. Plant Mol. Biol. 52: 331-341.

Brandstatter I and Kieber JJ (1998). Two genes with similarity to bacterial response regulators are rapidly and specifically induced by cytokinin in Arabidopsis. Plant Cell 10: 1009-1019.

Gan and Amansino (1995). Inhibition of leaf senescence by autoregulated production of cytokinin. Science 270: 1986-1988.

Giulini A, Wang J and Jackson D (2004). Control of phyllotaxy by the cytokinin-inducible response regulator homologue ABPHYL1. Nature 430: 1031-1034.

Higuchi M, Pischke MS, Mahonen AP, Miyawaki K, et al. (2004). In planta functions of the Arabidopsis cytokinin receptor family. Proc. Natl. Acad. Sci. U. S. A. 101: 8821-8826.

Hirose N, Makita N, Kojima M, Kamada-Nobusada T, et al. (2007). Overexpression of a type-A response regulator alters rice morphology and cytokinin metabolism. Plant Cell Physiol. 48: 523-539.

Hirose N, Takei K, Kuroha T, Kamada-Nobusada T, et al. (2008). Regulation of cytokinin biosynthesis, compartmentalization and translocation. J. Exp. Bot. 59: 75-83.

Hutchison CE, Li J, Argueso C, Gonzalez M, et al. (2006). The Arabidopsis histidine phosphotransfer proteins are redundant positive regulators of cytokinin signaling. Plant Cell 18: 3073-3087.

Hwang I and Sheen J (2001). Two-component circuitry in Arabidopsis cytokinin signal transduction. Nature 413: 383-389.

Hwang I, Chen HC and Sheen J (2002). Two-component signal transduction pathways in Arabidopsis. Plant Physiol. 129: 500-515.

Imamura A, Hanaki N, Umeda H, Nakamura A, et al. (1998). Response regulators implicated in His-to-Asp phosphotransfer signaling in Arabidopsis. Proc. Natl. Acad. Sci. U.S.A. 95: 2691-2696.

Inoue T, Higuchi M, Hashimoto Y, Seki M, et al. (2001). Identification of CRE1 as a cytokinin receptor from Arabidopsis. Nature 409: 1060-1063.

Ito Y and Kurata N (2006). Identification and characterization of cytokinin-signalling gene families in rice. Gene 382: 57-65. Jain M, Tyagi AK and Khurana JP (2006). Molecular characterization and differential expression of cytokinin-responsive type-A response regulators in rice (Oryza sativa). BMC Plant Biol. 6: 1.

Kakimoto T (1996). CKI1, a histidine kinase homolog implicated in cytokinin signal transduction. Science 274: 982-985.

Kakimoto T (2003). Perception and signal transduction of cytokinins. Annu. Rev. Plant Biol. 54: 605-627.

Kiba T, Yamada H, Sato S, Kato T, et al. (2003). The type-A response regulator, ARR15, acts as a negative regulator in the cytokinin-mediated signal transduction in Arabidopsis thaliana. Plant Cell Physiol. 44: 868-874.

Lee DJ, Park JY, Ku SJ, Ha YM, et al. (2007). Genome-wide expression profiling of Arabidopsis response regulator 7 (ARR7) overexpression in cytokinin response. Mol. Genet. Genomics 277: 115-137.

Lohrmann J and Harter K (2002). Plant two-component signaling systems and the role of response regulators. Plant Physiol. 128: 363-369.

Lohrmann J, Buchholz G, Keitel C, Sweere U, et al. (1999). Differential expression and nuclear localization of response regulator-like proteins from Arabidopsis thaliana. Plant Biol. 5: 495-505.

Miller CO, Skoog F, Von Saltza MH and Strong F (1955). Kinetin, a cell division factor from deoxyribonucleic acid. $J$. 
Am. Chem. Soc. 77: 1392.

Mok DW and Mok MC (2001). Cytokinin metabolism and action. Annu. Rev. Plant Physiol. Plant Mol. Biol. 52: 89-118. Muller B and Sheen J (2007). Advances in cytokinin signaling. Science 318: 68-69.

Nishimura C, Ohashi Y, Sato S, Kato T, et al. (2004). Histidine kinase homologs that act as cytokinin receptors possess overlapping functions in the regulation of shoot and root growth in Arabidopsis. Plant Cell 16: 1365-1377.

Osakabe Y, Miyata S, Urao T, Seki M, et al. (2002). Overexpression of Arabidopsis response regulators, ARR4/ATRR1/ IBC7 and ARR8/ATRR3, alters cytokinin responses differentially in the shoot and in callus formation. Biochem. Biophys. Res. Commun. 293: 806-815.

Porra RJ, Thompson WA and Kriedemann PE (1989). Determination of accurate extinction coefficients and simultaneous equations for assaying chlorophylls a and b extracted with four different solvents: verification of the concentration of chlorophyll standards by atomic absorption spectroscopy. Biochim. Biophys. Acta 975: 384-394.

Sakai H, Aoyama T, Bono H and Oka A (1998). Two-component response regulators from Arabidopsis thaliana contain a putative DNA-binding motif. Plant Cell Physiol. 39: 1232-1239.

Sakai H, Aoyama T and Oka A (2000). Arabidopsis ARR1 and ARR2 response regulators operate as transcriptional activators. Plant J. 24: 703-711.

Sakakibara H, Suzuki M, Takei K, Deji A, et al. (1998). A response-regulator homologue possibly involved in nitrogen signal transduction mediated by cytokinin in maize. Plant J. 14: 337-344.

Sentoku N, Sato Y and Matsuoka M (2000). Overexpression of rice OSH genes induces ectopic shoots on leaf sheaths of transgenic rice plants. Dev. Biol. 220: 358-364.

Stock AM, Robinson VL and Goudreau PN (2000). Two-component signal transduction. Annu. Rev. Biochem. 69: 183-215.

Suzuki T, Imamura A, Ueguchi C and Mizuno T (1998). Histidine-containing phosphotransfer (HPt) signal transducers implicated in His-to-Asp phosphorelay in Arabidopsis. Plant Cell Physiol 39: 1258-1268.

Suzuki T, Miwa K, Ishikawa K, Yamada H, et al. (2001). The Arabidopsis sensor His-kinase, AHk4, can respond to cytokinins. Plant Cell Physiol. 42: 107-113.

Taniguchi M, Kiba T, Sakakibara H, Ueguchi C, et al. (1998). Expression of Arabidopsis response regulator homologs is induced by cytokinins and nitrate. FEBS Lett. 429: 259-262.

Ueguchi C, Koizumi H, Suzuki T and Mizuno T (2001a). Novel family of sensor histidine kinase genes in Arabidopsis thaliana. Plant Cell Physiol. 42: 231-235.

Ueguchi C, Sato S, Kato T and Tabata S (2001b). The AHK4 gene involved in the cytokinin-signaling pathway as a direct receptor molecule in Arabidopsis thaliana. Plant Cell Physiol. 42: 751-755.

Urao T, Yakubov B, Yamaguchi-Shinozaki K and Shinozaki K (1998). Stress-responsive expression of genes for twocomponent response regulator-like proteins in Arabidopsis thaliana. FEBS Lett. 427: 175-178.

Werner T, Motyka V, Laucou V, Smets R, et al. (2003). Cytokinin-deficient transgenic Arabidopsis plants show multiple developmental alterations indicating opposite functions of cytokinins in the regulation of shoot and root meristem activity. Plant Cell 15: 2532-2550.

West AH and Stock AM (2001). Histidine kinases and response regulator proteins in two-component signaling systems. Trends Biochem. Sci. 26: 369-376.

Yonekura-Sakakibara K, Kojima M, Yamaya T and Sakakibara H (2004). Molecular characterization of cytokinin-responsive histidine kinases in maize. Differential ligand preferences and response to cis-zeatin. Plant Physiol. 134: 1654-1661. 